\title{
Milk-run routing problem with progress-lane in the collection of automobile parts
}

\author{
Zhaofang Mao · Dian Huang · Kan Fang · \\ Chengbo Wang · Dandan Lu
}

Received: date / Accepted: date

\begin{abstract}
In recent years, the automotive industry has faced an unprecedented crisis. In particular, the zero-inventory approach, which has been widely pursued by many automobile companies, seems to be impractical in some real production contexts since it requires an inventory of all parts but in low amounts. In this paper, we investigate a new logistics method which collects automobile parts by integrating the progress-lane (P-LANE) into the corresponding vehicle routing problem. We propose a mixed integer programming formulation for this new model, which can simultaneously determines the trip routes to collect automobile parts, as well as the P-LANE that each collected part should be assigned to, so as to minimize the total costs of the production and inbound logistics. The comparison with the zero-inventory model shows that the use of the P-LANE within the milk-run system could significantly decrease the total costs and also improve the transportation efficiency. To be specific, for small and large size instances, the total costs of the zero-inventory model are about $10 \%$ and $30 \%$ higher than the ones with P-LANE, respectively, which suggests that the periodic part collection model with P-LANE could be more appropriate for automobile manufacturing.
\end{abstract}

Keywords Milk-run system $\cdot$ Vehicle routing problem $\cdot$ Progress-lane $\cdot$ Lean logistics

This research was supported by the National Natural Science Foundation Council of China under projects 71872125 and 71701144 , and the Ministry of Science and Technology of China under project 2016IM030200.

Z. Mao

College of Management and Economics, Tianjin University, Tianjin 300072, China

D. Huang

College of Management and Economics, Tianjin University, Tianjin 300072, China

K. Fang

Corresponding author. College of Management and Economics, Tianjin University, Tianjin 300072, China

Tel.: +86-15602073837

E-mail: kfang@tju.edu.cn

C. Wang

Business School, Oxford Brookes University, Oxford OX3 0BP, UK

D. $\mathrm{Lu}$

College of Management and Economics, Tianjin University, Tianjin 300072, China 


\section{Introduction}

Under the current automobile manufacturing environment, many companies tend to emphasize several key stages of the supply chain, i.e., suppliers, manufacturers, distributors, and customers, as well as their coordination. In particular, the coordination of the production and distributors has been more widely discussed than that of the production and suppliers. In this work, we mainly focus on the coordination of scheduling decisions involving the production and inbound transportation in an automotive company, motivated by the practice of a Toyota Company in Tianjin.

As we know, the Toyota Company is famous for its Toyota Production System (TPS), which has been widely used to pursue zero-inventory. However, in practice, the goal of pursuing zero-inventory is usually difficult to achieve, therefore some other inventory modes have to be included in the real Toyota manufacturing plant. Among them, the progress-lane (P-LANE) is a very representative mode which stores all the parts needed for the production of the factory per day, as a terminal buffer to reduce the total costs and improve the transportation efficiency. Due to the fast turnover, the incoming parts will be usually used up within 4 hours.

As shown in Figure 1, P-LANE is an important cross-dock that connects the inbound logistics with outbound logistics. P-LANE converts the regular and non-quantitative outbound logistics into quantitative and irregular supply to the production line. In actual production, P-LANE generally contains 32 chains, which are used to coordinate the progress of the use of all parts in manufacturer's one-day production. The parts collected from the suppliers are assigned to P-LANE according to purchase orders after the parts are unloaded, and then evenly distributed to different number of P-LANEs according to the frequency of the logistics. Finally, the parts in each P-LANE are taken to the assembly line sequentially.

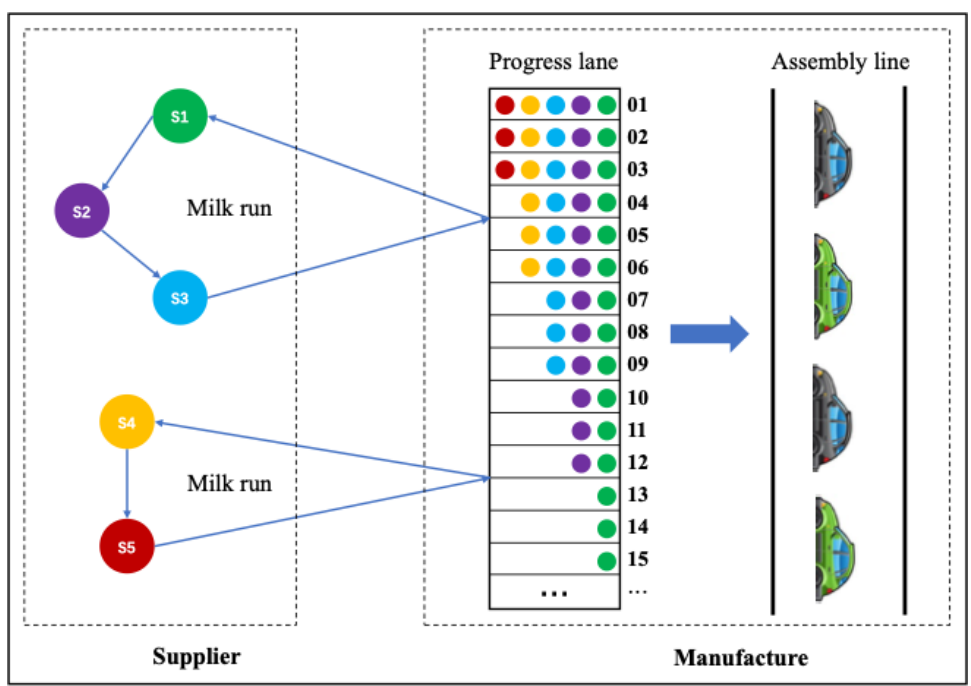

Fig. 1 Collection process of parts with P-LANE

To illustrate, Figure 2 shows an example of the parts transfer flow with P-LANE, in which the cargo truck transports the boxes containing the parts of suppliers A and B to the 
manufacturer by milk-run. Each box will have a specific order number. X1, X2, X3 and Y1, Y2, Y3 represent different parts of the suppliers. After arriving at the manufacturing plant, the parts will be divided into several P-LANEs according to the order number of the box and the frequency of logistics. Boxes with the same order tail number will be sent to the same numbered P-LANE. Different parts in one P-LANE are transported to different areas of the assembly line based on the production takt.

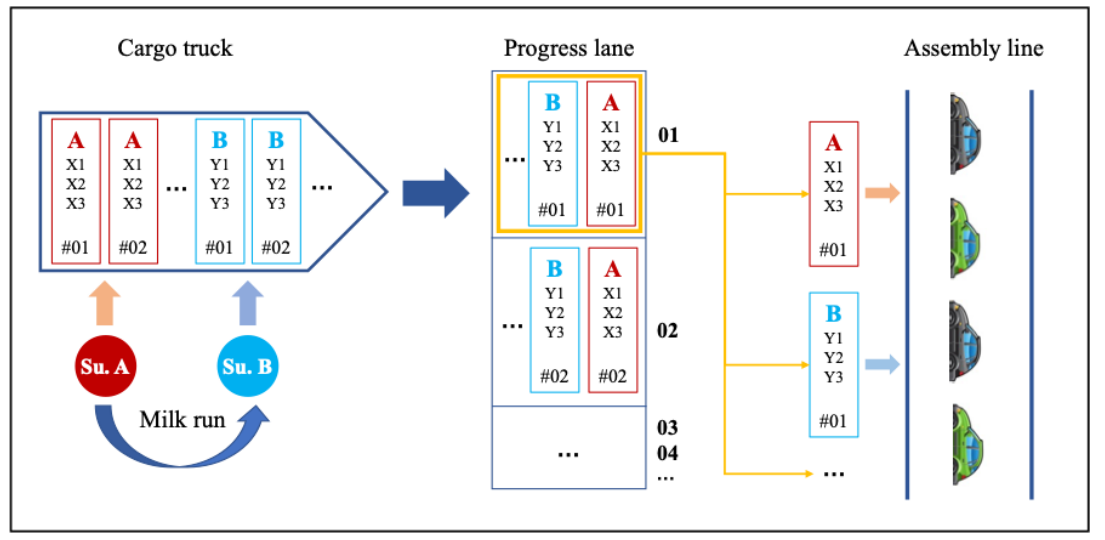

Fig. 2 Parts transfer flow with P-LANE

During the part collection and automobile manufacturing process, P-LANE has three significant effects: (1) Make parts available with the production schedule. It can prevent spillage and owing of parts in the plant, and thus reduce both the inventory costs and the shut down risks; (2) Make the milk-run more efficient. Suppliers are allowed to be more flexible in determining the quantity of parts supplied per truck. For example, the suppliers can increase the amount of each supply so as to avoid excessive frequency of milk-run and also save the transportation costs. (3) Realize high frequency and small volume supply of on-site parts. This way, the progress difference of inbound and outbound logistics can be absorbed to ensure smooth production of the Toyota Company.

However, through our investigation, we found that the Toyota company mainly makes their decisions on the route trips and scheduling times of part collection by practical experience, that is, there still exists opportunities for further improvement by using the mathematical modeling method.

As we know, the milk-run logistics is a method of parts collection in which the user (i.e., car assembly manufacturer) dispatches one truck at a specified time to visit various suppliers, following a predefined route to collect parts or products, and deliver them to the factory [Sadjadi et al., 2009, Chuah and Yingling, 2005]. In the production stage, given a monthly demand, we can accordingly determine the daily orders. Then, the assembly line starts working in a way given by the scheduled plans. To fulfill the demand from the assembly line, parts collected by the milk-run logistics are carried into the host terminal. After the collection, the orders are divided and placed according to each P-LANE. The time windows of different lanes cannot overlap. In addition, the orders in one lane cannot start to be produced unless all the components belonging to it have been delivered within the required time windows. Hence, it is necessary to study the production and inbound logistics simultaneously. In this work, we assume that the daily demand assigned to each P-LANE 
is given before the schedule, which is reasonable in the current automible manufacturing. We integrate the P-LANE into the milk-run routing and production process problem, and propose a mixed integer linear programming (MILP) model to improve the overall coordination. For simplicity, in this paper, we refer to this problem as the Milk-run routing problem with progress lane (or MRPPL for short), and call the corresponding MILP model for the MRPPL as the P-LANE model.

During the collection process, products from different orders with different suppliers can be collected together when the suppliers are not located far from each other. In this paper, we focus on a collection model with high frequency and small volume, which closely corresponds to the TPS. In the automotive industry, one vehicle consists of thousands of components, many of which are different for each color, engine size, and body style. Thus, holding each kind of the components at a safe level of stock is expensive. To simultaneously achieve just-in-time (JIT) and reduce the inventory costs, the zero-inventory model was presented and has become popular in some manufactures. Under the zero-inventory model, it is necessary to replenish the production line with higher frequency. In addition, the risk of a stopped line is high with the zero-inventory model, which may lead to large amount of penalty costs. In this paper, we compare the P-LANE model with the zero-inventory model to illustrate the advantages of P-LANE, and further show that a periodic part collection model combined with P-LANE could be more appropriate for automobile manufacturing.

The proposed work has the following contributions. First, to the best of our knowledge, we are one of the first to propose a mixed integer linear programming model that integrates the milk-run logistics and the P-LANE approach used in the Toyota factory together. Second, to solve the MRPPL, we use both the MILP optimization solver and the genetic algorithm to illustrate the advantage of the P-LANE model compared with the zero-inventory model. More importantly, the current application of the P-LANE and collection plan in the Toyota factory are mainly based on previous practical experience rather than precise quantitative solution methods and tools. This paper will be of great significance for reference to practical operations. From the perspective of the supply chain, the P-LANE model we proposed can be used by a supplier to arrange its own production and inventory so that the whole supply chain can operate more smoothly.

\section{Literature review}

As we mentioned above, increasingly more studies have focused on supply chain management. However, supply chain problems are usually difficult to model and analyze since the decisions at one stage are closely linked to all the other related stages. Hence, the coordination of the different key stages of the supply chain is needed to reduce both the inventory and transportation costs. Some researchers have focused their studies on particular strategies or policies when considering inventory costs [Lapierre et al., 2004], such as the zero-inventory management policy [Bramel and Simchi-Levi, 1995], the direct shipping policies [Gallego and Simchi-Levi, 1990], the power of two strategies [Federgruen et al., 1992], and the modified policy [Qu et al., 1999].

Many researchers have concentrated their studies on different combinations of supply chain management elements. In particular, the integrated production and distribution scheduling problems have aroused wide interests in recent years. Some studies discussed the problem only at strategic and tactical levels rather than at a practical level [Sarmiento and Nagi, 1999, Bilgen and Ozkarahan, 2004], while some others mainly focused on formulating different models and solving these models with various algorithms. Gao et al. [2015] 
considered a coordinated production and delivery problem in which orders are operated in batches considering limited vehicle capacity, with the condition of no-time-wait between the production and distribution. Li et al. [2015] investigated the integration of production and delivery problem on parallel batching machines. Gendreau et al. [2006] used neighborhood search heuristics to optimize the planned routes of vehicles in the context where new requests with a pick-up and a delivery location occur in real time. Sitek and Wikarek [2017] presented an optimization model implemented in hybrid environments that contain constraint logic programming, mathematical programming, and metaheuristics for the capacitated vehicle routing problem with pick-up and alternative delivery. However, in the automotive industry considered in our study, the assembly processes of different cars are almost the same and the production of cars is different from other kinds of products that have many production lines and high set-up costs. Hence, we are more concerned with the coordination between the production and inbound logistics. Most importantly, the Toyota Company has continuously pursued heijunka (leveling), causing the cycle time of various cars to be the same. Despite their diversity, some concepts and solutions proposed for the above coordination of production and delivery problems can also be used in our study.

Several researchers have also investigated the integration of production and inbound logistics. Among these work, most efforts mainly focused on balancing the transportation and inventory costs from the viewpoint of decreasing inventory. Berman and Wang [2006] considered the problem of selecting transportation strategies to analyze the trade-off between transportation and inventory cost. Lapierre and Ruiz [2007] presented a creative approach to improve the hospital logistics by coordinating the procurement and distribution operations with specific inventory capacities. Moreover, studies about collecting waste from a set of consumers [Benjamin and Beasley, 2010] and collecting oil from different cities [Lahyani et al., 2015] have exerted an important impact on theory and practice. In addition, Henke et al. [2015] discussed a capacitated vehicle routing problem that occurs in the context of glass waste collection. Chen et al. [2017] proposed a two-stage location-routing-inventory problem with time windows for food products. Nguyen et al. [2017] considered two-tiered city logistics systems accounting for both the inbound and outbound traffic. Montoya-Torres et al. [2015] provided a comprehensive review on different approaches used for the multidepot vehicle routing problems, and identified the most commonly considered operational constraints.

However, as a special case of the vehicle routing problem, the milk-run has become increasingly more popular in manufacturing [Kilic et al., 2012, Boysen et al., 2015]. The Toyota factory in Tianjin uses the milk-run combined with P-LANE to support its TPS. Currently, in the Toyota factory, there has many assembly plants located closely together and thus the company can use the milk-run system to collect parts from multiple suppliers and deliver them to the plants at the same time.

Until recently, research on using the milk-run to reduce the transportation cost and the vehicle waste in manufacturing is rather sparse and mostly focused on the theoretical aspect. Alnahhal et al. [2014] investigated the general framework of the decisions in in-plant milk run systems, and also the other related decisions that affect or are affected by milk run systems. Du et al. [2007] investigated the parameter settings of a real-time vehicle-dispatch system for consolidating the milk-run. Sadjadi et al. [2009] proposed a mathematical model for a special case of the auto industry and introduced several milk-run systems. Jiang et al. [2010] provided an optimization-based approach to satisfy high-frequency, small quantity deliveries. Brar and Saini [2011], who reviewed the literature on milk-run logistics and presented an overview of its implementation practices adopted by manufacturing organizations. Hosseini et al. [2014] developed a novel integer programming model to transport goods in a 
consolidation network and proposed a hybrid method of harmony search and simulated annealing based heuristics to solve their problem. Patel et al. [2014] reviewed the most efficient methods to solve the problem of milk run material supply system in vehicle routing problem with simultaneous pickup and delivery. Staab et al. [2016] presented a generic model to simulate different milk-run systems, focusing on the physical handling steps. In addition, there are very few studies on the combination of milk-run and P-LANE. The only work that we can find is the one by Nemoto et al. [2010], in which the authors considered the cooperation of milk-run and P-LANE in the Toyota Thailand factory, and showed that gaps between transport frequency and production leveling could be reduced, and better production process could be achieved.

The above studies show that modeling the supply and production problem within the milk-run logistics systems for the automotive industry has not yet been done. We fulfill this need in an innovative way in this paper. The supplier, inventory, and production stages are considered simultaneously. In particular, it is the first time that P-LANE used by the Toyota Company was applied when modeling the problem as a mixed integer program. In particular, there are usually many suppliers which are located close to the car manufacturer, which makes the integrated supply and production problem complex. Consequently, it is worth using optimization-based methods to solve this practical problem, which is a matter of urgent concern for the Toyota factory in Tianjin.

\section{Problem formulation}

Our study is motivated by the practice of the Toyota Company and aims to solve the MRPPL, an extension of the milk-run routing problem that considers P-LANE, in a quantitative, lowcost, and high-quality way. The MRPPL is described as follows: Let $G=(\mathscr{V}, \mathscr{A})$ be an undirected complete graph, where $\mathscr{V}=\{0,1, \ldots, n\}$ is the vertex set and $\mathscr{A}$ is the arc set. Vertices $i=1, \ldots, n$ is the set of suppliers and vertex 0 corresponds to the manufacturer. Obviously, when we collect the parts directly from each supplier, then the maximum number of routes is $n$, thus we define $\mathscr{R}=\{1, \ldots, n\}$ as the set of routes. In addition, $\mathscr{P}=\{1, \ldots, m\}$ is the set of P-LANE. We are given the following inputs: $c_{j k}$ is the nonnegative cost between any two vertices $j, k \in \mathscr{V}$, and $\phi$ is the transportation cost per unit distance. $b_{j p}$ is the number of boxes needed from supplier $j \in \mathscr{V} \backslash\{0\}$ on P-LANE $p \in \mathscr{P}$, and $b_{j}=\sum_{p \in \mathscr{P}} b_{j p}$ is the total number of boxes needed from supplier $j$ in manufacturer's one-day production. $\eta_{j s}$ is the average number of boxes collected from supplier $j$ per each visit if supplier $j$ is visited $s$ times; $d_{p}$ is the due date of P-LANE $p$, the earliness and tardiness penalty costs per unit time are $\theta$ and $\psi$ respectively, and $\omega$ is the fixed cost per vehicle per visit. $U_{j}$ is the unloading time per box for the parts collected from supplier $j$. Moreover, we assume that all the vehicles are identical with a capacity $Q$, and the available number of vehicles in the manufacturer is sufficiently large. It is easy to see that the maximum visiting frequency of each route is at most $\sum_{j} b_{j} / Q$, and we define $\mathscr{F}=\left\{1, \ldots,\left\lceil\sum_{j} b_{j} / Q\right\rceil\right\}$ as the set of visiting frequencies for each route. The objective of the MRPPL is to find a feasible route trip plan as well as an associated scheduling plan for each route so as to minimize the total costs.

In this section, we propose a mixed integer linear program for the MRPPL. We define the following decision variables:

- $x_{r}$ is equal to 1 if route $r \in \mathscr{R}$ is used to collect parts from suppliers and 0 otherwise;

$-y_{j r}$ is equal to 1 if supplier $j$ is assigned to route $r$ and 0 otherwise;

$-z_{j k r}$ is equal to 1 if arc $(j, k)$ is visited by route $r$, and 0 otherwise;

$-u_{r s}$ is equal to 1 if the frequency of route $r$ is $s$ and 0 otherwise; 
- $D_{r s}$ is the departure time of the $s$ th visit from the manufacturer for route $r$;

- $A_{r s}$ is the arrival time of the sth visit to the manufacturer for route $r$;

- $\sigma_{j r s}$ is equal to 1 if $y_{j r}=1, u_{r s}=1$ and 0 otherwise;

- $\delta_{j k r s}$ is equal to 1 if $z_{j k r}=1, u_{r s}=1$ and 0 otherwise;

- $\xi_{j r s t p}$ is equal to 1 if $\sigma_{j r s}=1$ and the $t$ th visit of route $r$ meet the demand needed from supplier $j$ on P-LANE $p$ and 0 otherwise;

- $F_{j p}$ is the finish time when the parts collected from supplier $j$ meet the demand on PLANE $p$;

- $E_{j p}$ is the earliness of supplier $j$ on P-LANE $p$, i.e., $E_{j p}=\max \left\{0, d_{p}-F_{j p}\right\}$;

- $T_{j p}$ is the tardiness time of supplier $j$ on P-LANE $p$, i.e., $T_{j p}=\max \left\{0, F_{j p}-d_{p}\right\}$.

Let $M$ be a sufficiently large number, then the MRPPL can be modeled as the following optimization model (1), i.e., the P-LANE model.

minimize $\sum_{j \in \mathscr{V}} \sum_{k \in \mathscr{V}} \sum_{r \in \mathscr{R}} \sum_{s \in \mathscr{F}} \phi s c_{j k} \delta_{j k r s}+\sum_{r \in \mathscr{R}} \sum_{s \in \mathscr{F}} \omega s u_{r s}+\sum_{j \in \mathscr{V} \backslash\{0\}} \sum_{p \in \mathscr{P}} \theta E_{j p}+\sum_{j \in \mathscr{V} \backslash\{0\}} \sum_{p \in \mathscr{P}} \psi T_{j p}$

subject to

$x_{r+1} \leq x_{r}$ for $r \in \mathscr{R}$

$\sum_{r \in \mathscr{R}} y_{j r}=1 \quad$ for $j \in \mathscr{V} \backslash\{0\}$;

$\sum_{j \in \mathscr{V} \backslash\{0\}} y_{j r} \geq x_{r} \quad$ for $r \in \mathscr{R}$

$\sum_{k \in \mathscr{V}} z_{j k r}=y_{j r} \quad$ for $j \in \mathscr{V} \backslash\{0\} ; r \in \mathscr{R}$;

$\sum_{j \in \mathscr{V}} z_{j k r}=y_{k r} \quad$ for $k \in \mathscr{V} \backslash\{0\} ; r \in \mathscr{R}$;

$\sum_{(j, k) \in S \times S} z_{j k r} \leq|S|-1 \quad$ for $S \subset \mathscr{V} \backslash\{0\},|S| \geq 2 ; r \in \mathscr{R} ;$

$\sum_{s \in \mathscr{F}} u_{r s}=x_{r} \quad$ for $r \in \mathscr{R}$

$\sum_{j \in \mathscr{N} \backslash\{0\}} b_{j} y_{j r} \leq \sum_{s \in \mathscr{F}} s Q u_{r s} \quad$ for $r \in \mathscr{R} ;$

$\sum_{j \in \mathscr{V} \backslash\{0\}} b_{j} y_{j r} \geq \sum_{s \in \mathscr{F}}[(s-1) Q+1] u_{r s} \quad$ for $r \in \mathscr{R} ;$

$2 \delta_{j k r s} \leq z_{j k r}+u_{r s} \quad$ for $j, k \in \mathscr{V}, r \in \mathscr{R}, s \in \mathscr{F}$;

$1+\delta_{j k r s} \geq z_{j k r}+u_{r s} \quad$ for $j, k \in \mathscr{V}, r \in \mathscr{R}, s \in \mathscr{F}$;

$2 \sigma_{j k r s} \leq y_{j r}+u_{r s} \quad$ for $j \in \mathscr{V} \backslash\{0\}, r \in \mathscr{R}, s \in \mathscr{F}$;

$1+\sigma_{j k r s} \geq y_{j r}+u_{r s} \quad$ for $j \in \mathscr{V} \backslash\{0\}, r \in \mathscr{R}, s \in \mathscr{F}$;

$\sum_{t=1}^{s} t \eta_{j s} \xi_{j r s t} \geq \sum_{k=1}^{p} b_{j k} \sigma_{j r s} \quad$ for $j \in \mathscr{V} \backslash\{0\}, r \in \mathscr{R}, s \in \mathscr{F}, p \in \mathscr{P}$;

$\sum_{t=1}^{s}\left[(t-1) \eta_{j s}+1\right] \xi_{j r s t} \leq \sum_{k=1}^{p} b_{j k} \sigma_{j r s} \quad$ for $j \in \mathscr{V} \backslash\{0\}, r \in \mathscr{R}, s \in \mathscr{F}, p \in \mathscr{P} ;$

$\sum_{t=1}^{s} \xi_{j r s t p}=\sigma_{j r s} \quad$ for $j \in \mathscr{V} \backslash\{0\}, r \in \mathscr{R}, s \in \mathscr{S}, p \in \mathscr{P}$; 
$T_{j p} \geq F_{j p}-d_{p}$ for $j \in \mathscr{V} \backslash\{0\}, p \in \mathscr{P}$;

The objective (1a) minimizes the total costs, including the variable and fixed transportation costs, and the earliness and tardiness costs. Constraints (1b) ensure that route $r+1 \mathrm{can}$ be opened to collect parts only if route $r$ has been used. Constraints (1c) ensure that each supplier can be visited by exactly one route. Constraints (1d) specifies that there must exist at least one supplier on any opened route. Constraints (1e) and (1f) establish the relationship between variables $y$ and $z$, which also ensure that the flow in each route is conserved. Constraints (1g) eliminates all possible subtours. Constraints (1h)-(1j) ensure that each route can have exactly one frequency and also determine the frequency of each route, once the assignment of suppliers to the routes is given. Constraints (1k)-(1n) ensure that the variables $\delta$ and $\sigma$ take their intended values. Constraints (1o)-(1q) establish the relationship between variables $\sigma$ and $\xi$. Constraints (1r) gives the relationship between the departure time and arrival time of each visit for each route. Constraints (1s) and (1t) ensure that the completion time of all kinds of parts are coordinated with the whole P-LANE. Constraints (1u) and (1v) define the earliness and tardiness time.

The MILP model proposed above provides a compact and unambiguous specification of the problem. We use it when comparing the P-LANE model with the zero-inventory models to analyze the performance of P-LANE. In fact, only small instances can be solved optimally by commercial optimization solvers. This is not surprising because even the classic VRP is already a hard problem. Most importantly, the key point of this paper is to introduce the P-LANE model to various manufacturers and other researchers who are interested in lean inbound logistics.

\section{Algorithm design}

In this study, we use Gurobi Optimizer and the Python programming language to code and solve the MILP model for small instances, and develop a genetic algorithm using Matlab language to obtain approximate solutions of the MRPPL within a reasonable computational time for large size instances. In what follows, we provide the main procedures which are used to implement our genetic algorithm.

\subsection{Chromosome representation}

In a genetic algorithm, a solution is represented by a chromosome composed of several genes. For vehicle routing problems, a permutation is often used where genes act as the supplier location and the order of the genes indicates the sequence of the supplier locations that are visited [El Fallahi et al., 2008]. In the MRPPL, all suppliers are divided into different routes. Therefore, a chromosome should not only provide the set of suppliers of each route but also the visiting sequence in each route. 
To illustrate the chromosome that we used to represent the solutions, we consider the following example. Suppose there are 10 suppliers in the MRPPL, which are divided into 4 subgroups: $\{2,4,1\},\{7,9\},\{6,5,8\},\{3,10\}$. The sequence of each subgroup indicates the order of suppliers to be visited in each route, that is, the first subgroup presents a trip of " $0 \rightarrow 2 \rightarrow 4 \rightarrow 1 \rightarrow 0$ ". Then this solution can be represented by the chromosome as shown in Figure 3, in which the first part includes 10 elements that is combined by the sequences of all the routes, and the second part includes 3 elements, which indicates that the number of subgroups will be $3+1=4$. In addition, the value of each element in the second part indicates the breaking points by which suppliers are divided into different subgroups. For example, the first elements " 3 " in the second part indicates that the first three elements, i.e., $\{2,4,1\}$ will form a subgroup, and the second element "5" indicates that the elements in the fourth and fifth positions, i.e., $\{7,9\}$, will form another subgroup.

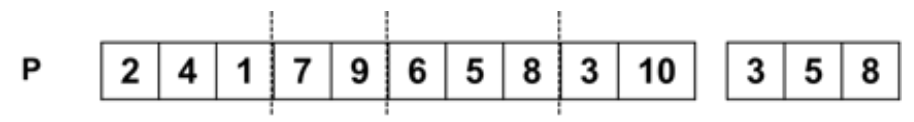

Fig. 3 A chromosome for the solution with subgroups $\{2,4,1\},\{7,9\},\{6,5,8\},\{3,10\}$.

Since our objective is to minimize the total costs, therefore the lower the total costs we obtained by the solution, the better the solution is. As we mentioned, in the MRPPL, the total costs contains four components, i.e., the transportation cost, the fixed cost, the earliness and tardiness costs. Let $Z$ be the total costs we obtained from the solution, then we define $f=1 / Z$ as the fitness value function.

\subsection{Initial population generation}

To generate an initial population, we randomly generate a permutation of all the $n$ suppliers, and set it as the first part of the chromosome. Then, we generate a random number $k \in$ $\{1, \ldots, n-1\}$ to divide the suppliers into $k+1$ subgroups. For the $k$ elements in the second part, some positions are then designated as the breaking points. This way, we generate an initial population shows which route each supplier belongs to and the sequence of suppliers in each route, as well as the total number of routes we used to collect parts from the suppliers.

\subsection{Selection}

To preserve the individuals that with better fitness values in the next generation, we use both of the following two actions to select individuals: the first one is to select a proportion of the better individuals for the next selection action, the other is to use the roulette wheel selection strategy.

\subsection{Crossover}

As a key step of the genetic algorithm, the crossover operators have been frequently studied in the literature and many methods are proposed to perform an exchange between two parents. Many well-known crossover operators like the intermediate, parent-centric, and 
unimodal normal distribution crossover operators are widely used. However, these classical crossover operators may generate solutions with infeasible permutations. Note that the route sequence in this paper is a permutation problem, therefore we apply an evolutionary crossover operator called the order crossover to complete this process [Talbi, 2009]: First, we randomly select two crossover points. From parent 1, we copy the part between the two points at the same absolute positions into the offspring. Then, we pick the elements that are not already selected from parent 1 , and fill them into the offspring starting from the second crossover points. Figure 4 shows an example of the order crossover operator.

P1

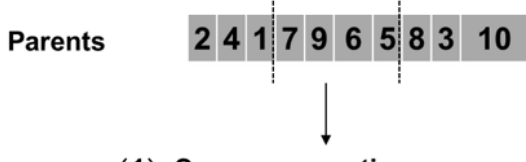

(1) Crossover section

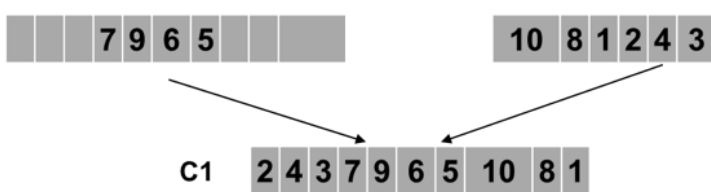

Fig. 4 An example of the order crossover operator for permutations

\subsection{Mutation}

Mutation is an auxiliary operation of the genetic algorithm that ensures the diversity of the species. In this paper, the swap operator is employed, which has been proven to be quite effective, that is, two elements are chosen randomly and replace each other in the first part (the supplier sequence) of the chromosomes.

\section{Case study}

In this part, we mainly focus on a practical case generated from Factory M, one of the wellknown factories of the FAW-Toyota Motor Co. Ltd, a joint-venture company established by FAW and Toyota in Tianjin. During the past two decades, Factory M has been striving for continuous improvement and lean production, and has made itself become a very important production base for FAW-Toyota. We compare the P-LANE model with the zero-inventory model for instances with different sizes, based on the real data we obtained from Factory M. The result shows that the P-LANE model could significantly reduce the total costs of inbound logistics and production on both small- and large-scale instances. 


\subsection{The zero-inventory model}

In the zero-inventory model, we divide Factory M's one-day production into multiple production cycles. To ensure comparability, the number of production cycles is the same as the number of P-LANEs. In each production cycle $p \in \mathscr{P}$, the demand needed from suppliers is defined as the demand of P-LANE $p$. Similar to the P-LANE model, we also assume that there are enough trucks and the parts arrive at the factory on time only when needed. Therefore, in the zero-inventory model, there are only fixed cost and transportation cost, without any earliness and tardiness costs. The production cycles are independent from each other and the optimal route for each production cycle is planned separately. As a result, for each production cycle $p \in \mathscr{P}$, the corresponding optimal solution by the zero-inventory model can be obtained by solving the following optimization model:

$$
\begin{array}{ll}
\text { minimize } & \sum_{j \in \mathscr{V}} \sum_{k \in \mathscr{V}} \sum_{r \in \mathscr{R}} \sum_{s \in \mathscr{F}} \phi s c_{j k} \delta_{j k r s}+\sum_{r \in \mathscr{R}} \sum_{s \in \mathscr{F}} \omega s u_{r s} \\
\text { subject to } & \text { Constraints (1b)- (1q), and (1w). }
\end{array}
$$

\subsection{Instances generation}

As explained before, the MRPPL is different from other vehicle routing problems or milkrun problems described in the literature because of its application for P-LANE. Therefore, there is no point in using previous instances used in literature for our experiments. Instead, we choose to generate some new problem instances as follows.

The capacity of the transport vehicle, the geographic locations of the suppliers, and the daily supply are all real data collected from Factory M. Each supplier's location is labelled by its longitude and latitude on Google Maps, and is transformed into the X and Y coordinates. The raw and processed coordinates of Factory $M$ and its 40 suppliers are given in the Appendix (see Table 8). Sample suppliers are randomly selected from all suppliers of Factory M. The average daily supply of suppliers in the instances and the average distance from Factory $\mathrm{M}$ are basically the same as the average data of all suppliers of Factory $\mathrm{M}$. In the small-scale instances, the number of P-LANEs is 4 , and the number of suppliers is $5 / 6 / 7$. In the large-scale instances, the number of P-LANEs is 8 , and the number of suppliers is 20/30/40. Figures 5 and 6 show the locations of suppliers for the small- and large-scale instances, respectively.

The cost coefficients of the transportation, fixed, earliness and tardiness costs used in our case study were guided by the cases found in most manufacturing companies as well as the theories obtained in many studies. The transportation cost coefficient was determined by the total transportation distances, which can also be viewed as the total transportation time. The fixed cost coefficient is positively related to transportation frequency. The earliness cost was defined according to the earliness time, which is the time that the parts are stored in a P-LANE waiting for production. Similarly, the tardiness cost is incurred if the parts cannot meet the demand of the production as scheduled, which could cause the assembly line to stop. Specifically, the coefficients of these four costs were set to $\phi=3, f=18, \theta=2$, and $\psi=4$ respectively. In addition, the data and values of the cost coefficients used in the zeroinventory model were identical to those in the P-LANE model. 


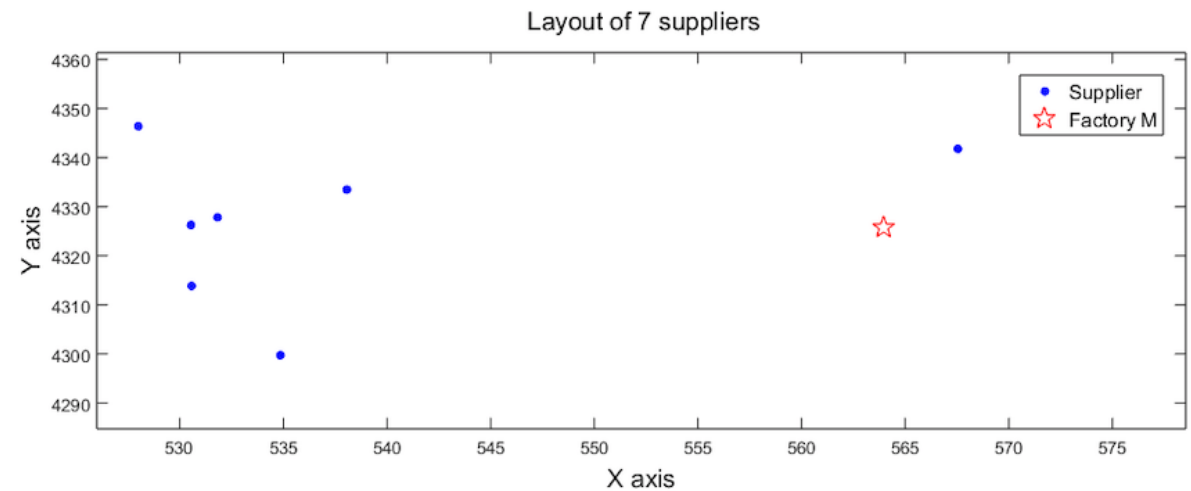

Fig. 5 Locations of the 7 suppliers in small-scale instances

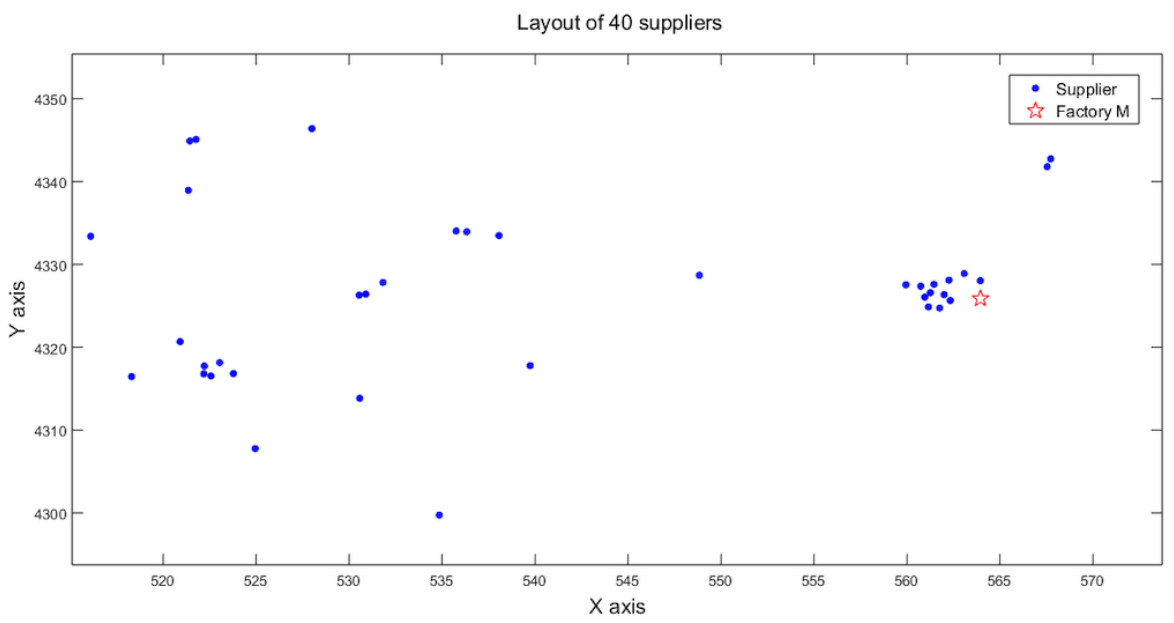

Fig. 6 Locations of the 40 suppliers in large-scale instances

\subsection{Experiments on small-scale instances}

For small-scale instances, we use Gurobi Optimizer to solve the MRPPL optimally. Tables 1 and 2 present the corresponding results of the routing and frequency by solving the P-LANE model and the zero-inventory model respectively.

From Tables 1 and 2, we can see that when the number of suppliers is small, i.e., $n=5,6$, the optimal routes generated by the two models are no much difference, and the supplier sequences of the zero-inventory model during each production cycle are also the same. However, when the number of suppliers reaches 7 , the routes of the two models start to show their differences, and the supplier sequence generated by the zero-inventory model during each production cycle also differentates itself from the others. The reason for such phenomenon may be that: when the number of suppliers increases, there are more flexible routing and scheduling plans that we can obtain to reduce the total costs.

For the purpose of illustration, we give the optimal routes and the visiting frequency of each route by the P-LANE model for the instance with 7 suppliers (see Figure 7). In this 
Table 1 Results of the routing and frequency by the P-LANE model for small-scale instances

\begin{tabular}{llll}
\hline \# Suppliers & Route & Supplier sequence & Frequency \\
\hline \multirow{5}{*}{5} & R1 & S0-S1-S3-S0 & 4 \\
& R2 & S0-S2-S0 & 4 \\
& R3 & S0-S4-S0 & 4 \\
& R4 & S0-S5-S0 & 5 \\
\hline \multirow{4}{*}{6} & R1 & S0-S1-S3-S0 & 4 \\
& R2 & S0-S2-S0 & 4 \\
& R3 & S0-S4-S0 & 4 \\
& R4 & S0-S5-S0 & 5 \\
& R5 & S0-S6-S0 & 2 \\
\hline \multirow{4}{*}{7} & R1 & S0-S1-S7-S0 & 4 \\
& R2 & S0-S2-S0 & 4 \\
& R3 & S0-S3-S6-S0 & 3 \\
& R4 & S0-S4-S0 & 4 \\
& R5 & S0-S5-S0 & 5 \\
\hline
\end{tabular}

Table 2 Results of the routing and frequency by the zero-inventory model for small-scale instances

\begin{tabular}{|c|c|c|c|c|c|c|c|c|c|}
\hline \multirow{2}{*}{ \# Suppliers } & \multirow{2}{*}{ Route } & \multicolumn{2}{|c|}{ Production Cycle 1} & \multicolumn{2}{|c|}{ Production Cycle 2} & \multicolumn{2}{|c|}{ Production Cycle 3} & \multicolumn{2}{|c|}{ Production Cycle 4} \\
\hline & & Supplier sequence & Frequency & Supplier sequence & Frequency & Supplier sequence & Frequency & Supplier sequence & Frequency \\
\hline \multirow{4}{*}{5} & R1 & S0-S1-S3-S0 & 1 & S0-S1-S3-S0 & 1 & S0-S1-S3-S0 & 1 & S0-S1-S3-S0 & \\
\hline & R2 & S0-S2-S0 & 1 & S0-S2-S0 & 1 & S0-S2-S0 & 1 & S0-S2-S0 & 1 \\
\hline & R3 & S0-S4-S0 & 1 & S0-S4-S0 & 1 & S0-S4-S0 & 1 & S0-S4-S0 & 1 \\
\hline & R4 & S0-S5-S0 & 2 & S0-S5-S0 & 2 & S0-S5-S0 & 2 & S0-S5-S0 & 2 \\
\hline \multirow{5}{*}{6} & R1 & S0-S1-S3-S0 & 1 & S0-S1-S3-S0 & 1 & S0-S1-S3-S0 & 1 & S0-S1-S3-S0 & 1 \\
\hline & R2 & S0-S2-S0 & 1 & S0-S2-S0 & 1 & S0-S2-S0 & 1 & S0-S2-S0 & 1 \\
\hline & R3 & S0-S4-S0 & 1 & S0-S4-S0 & 1 & S0-S4-S0 & 1 & S0-S4-S0 & 1 \\
\hline & R4 & S0-S5-S0 & 2 & S0-S5-S0 & 2 & S0-S5-S0 & 2 & S0-S5-S0 & 2 \\
\hline & R5 & S0-S6-S0 & 1 & S0-S6-S0 & 1 & S0-S6-S0 & 1 & S0-S6-S0 & 1 \\
\hline \multirow{5}{*}{7} & R1 & S0-S1-S7-S0 & 1 & S0-S2-S0 & 1 & S0-S1-S7-S0 & 1 & S0-S1-S3-S0 & 1 \\
\hline & R2 & S0-S2-S6-S0 & 2 & S0-S3-S6-S0 & 1 & S0-S2-S5-S0 & 2 & S0-S4-S0 & 1 \\
\hline & R3 & S0-S3-S0 & 1 & S0-S4-S1-S7-S0 & 2 & S0-S3-S6-S0 & 1 & S0-S5-S0 & 2 \\
\hline & R4 & S0-S4-S0 & 1 & S0-S5-S0 & 2 & S0-S4-S0 & 1 & S0-S6-S2-S7-S0 & 2 \\
\hline & R5 & S0-S5-S0 & 2 & - & - & - & - & - & - \\
\hline
\end{tabular}

-: No more route for the corresponding production cycle.

figure, the red star represents Factory M, and the blue squares are the suppliers. The larger the square is, the greater the daily supply will be. Different color lines represent different collection routes. The thicker the line is, the higher the collection frequency will be.

In Table 3, the bold value indicates the optimal value of each category among the two models. We can see that the P-LANE model could significantly reduce the total costs compared to the zero-inventory model.

Table 3 Comparison of the costs between the two models for small-scale instances

\begin{tabular}{lllllll}
\hline \# Suppliers & Model & Transportation cost & Fixed cost & Earliness cost & Tardiness cost & Total costs \\
\hline \multirow{2}{*}{5} & P-LANE model & $\mathbf{3 2 4 6}$ & $\mathbf{3 0 6}$ & 0 & 0 & $\mathbf{3 5 5 2}$ \\
& Zero-inventory model & 3552 & 360 & 0 & 0 & 3912 \\
\hline \multirow{2}{*}{6} & P-LANE model & $\mathbf{3 7 2 6}$ & $\mathbf{3 4 2}$ & 400 & 0 & $\mathbf{4 4 6 8}$ \\
& Zero-inventory model & 4512 & 432 & $\mathbf{0}$ & 0 & 4944 \\
\hline \multirow{2}{*}{7} & P-LANE model & $\mathbf{4 1 3 1}$ & $\mathbf{3 6 0}$ & 280 & 480 & $\mathbf{5 2 5 1}$ \\
& Zero-inventory model & 5346 & 432 & $\mathbf{0}$ & $\mathbf{0}$ & 5778 \\
\hline
\end{tabular}




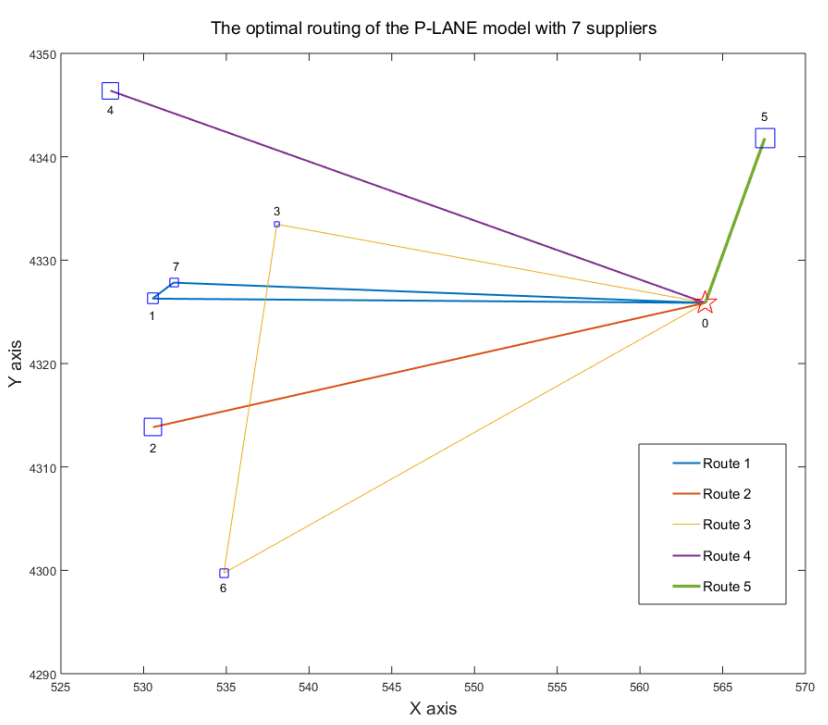

Fig. 7 The optimal routing of the P-LANE model with 7 suppliers

\subsection{Experiments on large-scale instances}

For large-scale instances, we use the proposed genetic algorithm to solve both the P-LANE and the zero-inventory models. Tables 4 and 5 present the corresponding results of the routing and frequency by the P-LANE and the zero-inventory models respectively.

Table 4 The routing and frequency results by the P-LANE model for large-scale instances

\begin{tabular}{lllllll}
\hline \multirow{2}{*}{ Route } & \multicolumn{2}{c}{ 20 Suppliers } & \multicolumn{2}{c}{ 30 Suppliers } & \multicolumn{2}{c}{ 40 Suppliers } \\
\cline { 2 - 7 } & Supplier sequence & Frequency & Supplier sequence & Frequency & Supplier sequence & Frequency \\
\hline R1 & S0-S1-S14-S9-S12-S0 & 10 & S0-S1-S10-S29-S11-S0 & 8 & S0-S2-S0 & 3 \\
R2 & S0-S2-S16-S0 & 4 & S0-S4-S3-S0 & 5 & S0-S3-S0 & 4 \\
R3 & S0-S4-S3-S0 & 5 & S0-S9-S28-S12-S0 & 9 & S0-S4-S29-S0 & 4 \\
R4 & S0-S6-S13-S5-S17-S0 & 4 & S0-S13-S8-S0 & 9 & S0-S5-S0 & 1 \\
R5 & S0-S10-S8-S15-S0 & 12 & S0-S15-S14-S0 & 7 & S0-S7-S20-S25-S0 & 8 \\
R6 & S0-S19-S18-S11-S0 & 13 & S0-S16-S5-S0 & 2 & S0-S9-S11-S0 & 4 \\
R7 & S0-S20-S7-S0 & 6 & S0-S18-S0 & 8 & S0-S12-S18-S0 & 9 \\
R8 & - & - & S0-S19-S0 & 5 & S0-S13-S0 & 2 \\
R9 & - & - & S0-S20-S7-S0 & 6 & S0-S14-S8-S16-S01 & 0 \\
R10 & - & - & S0-S21-S0 & 2 & S0-S15-S0 & 5 \\
R11 & - & - & S0-S23-S0 & 3 & S0-S19-S0 & 5 \\
R12 & - & - & S0-S24-S6-S0 & 4 & S0-S21-S0 & 2 \\
R13 & - & - & S0-S25-S26-S0 & 7 & S0-S22-S0 & 2 \\
R14 & - & - & S0-S27-S17-S0 & 3 & S0-S23-S6-S0 & 3 \\
R15 & - & - & S0-S30-S2-S22-S0 & 5 & S0-S24-S26-S0 & 10 \\
R16 & - & - & - & - & S0-S27-S17-S0 & 3 \\
R17 & - & - & - & - & S0-S28-S30-S1-S0 & 9 \\
R18 & - & - & - & - & S0-S31-S38-S0 & 2 \\
R19 & - & - & - & - & S0-S34-S36-S0 & 3 \\
R20 & - & - & - & - & S0-S35-S10-S0 & 8 \\
R21 & - & - & - & S0-S39-S0 & 7 \\
R22 & - & - & - & S0-S40-S37-S32-S33-S0 & 13 \\
\hline
\end{tabular}

-: No more route for the instance with the corresponding number of suppliers. 
Table 5 The routing and frequency results by the zero-inventory model for large-scale instances

\begin{tabular}{|c|c|c|c|}
\hline $\begin{array}{l}\text { Production } \\
\text { cycle }\end{array}$ & Supplier sequences with $n=20$ & Supplier sequences with $n=30$ & Supplier sequences with $n=40$ \\
\hline \multirow{8}{*}{1} & \# routes: 14; Total frequencies: 14; & \# routes: 21 ; Total frequencies: 22 ; & \# routes: $29 ;$ Total frequencies: 30 \\
\hline & S0-S20-S0; S0-S5-S0; S0-S6-S0; & S0-S12-S0; S0-S13-S0; S0-S23-S0; & S0-S12-S0; S0-S21-S33-S0; S0-S28-S0; S0-S5-S25-S0; \\
\hline & S0-S13-S0; S0-S19-S17-S0; & S0-S27-S0; $\underline{\text { S0-S26-S2-S21-S28-S0; }}$ & S0-S4-S0; S0-S36-S2-S0; S0-S26-S0; S0-S1-S0; \\
\hline & S0-S18-S0; S0-S2-S0; S0-S16-S3-S0; & S0-S8-S30-S0; S0-S7-S25-S5-S0; & S0-S10-S11-S0; S0-S8-S0; S0-S14-S0; S0-S3-S0; \\
\hline & S0-S4-S0; S0-S1-S15-S0; & S0-S15-S0; S0-S4-S0; S0-S14-S0; & SO-S40-S0; S0-S39-SO; SO-S13-S0; SO-S18-S0; \\
\hline & S0-S10-S9-S11-S14-S0; S0-S7-S0; & S0-S11-S0; S0-S10-S19-S0; S0-S9-S0; & S0-S15-S0; S0-S37-S0; S0-S24-S0; S0-S19-S0; \\
\hline & S0-S12-S0; S0-S8-S0; & S0-S16-S0; S0-S18-S17-S0; & S0-S9-S0;S0-S7-S17-S16-S0; S0-S31-S0; \\
\hline & & $\begin{array}{l}\text { S0-S22-S20-S0; S0-S1-S0; S0-S6-S0; } \\
\text { S0-S3-S0; S0-S24-S0; S0-S29-S0; }\end{array}$ & $\begin{array}{l}\text { S0-S34-S6-S0; S0-S32-S23-S0; S0-S30-S29-S0; } \\
\text { S0-S27-S20-S35-S0; S0-S38-S0; S0-S22-S0; }\end{array}$ \\
\hline \multirow{9}{*}{2} & \# routes: 14 ; Total frequencies: $14 ;$ & \# routes: $21 ;$ Total frequencies: $22 ;$ & \# routes: 29 ; Total frequencies: 31 \\
\hline & S0-S6-S0; S0-S4-S13-S10-S0; & S0-S18-S0; S0-S19-S25-S0; S0-S15-S0; & S0-S22-S0; S0-S16-S0; S0-S1-S0; S0-S9-S0; \\
\hline & S0-S3-S2-S0; S0-S18-S0; S0-S19-S0; & S0-S6-S0; S0-S20-S0; S0-S29-S0; & S0-S27-S20-S0; S0-S28-S0; S0-S35-S0; S0-S21-S0; \\
\hline & S0-S5-S14-S0; S0-S7-S15-S0; & S0-S28-S0; S0-S13-S0; S0-S4-S0; & S0-S7-S2-S0; S0-S36-S10-S0; S0-S40-S0; S0-S6-S0; \\
\hline & S0-S11-S0; S0-S8-S0; S0-S12-S20-S0; & S0-S16-S12-S0; S0-S5-S9-S0; & S0-S32-S0; S0-S23-S30-S0; S0-S12-S0; S0-S11-S0; \\
\hline & S0-S1-S0; S0-S16-S0; S0-S9-S0; & S0-S26-S17-S23-S0; S0-S27-S7-S0; & 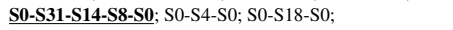 \\
\hline & S0-S17-S0; & S0-S22-S0; S0-S8-S0; S0-S11-S0; & 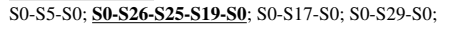 \\
\hline & & S0-S30-S0; S0-S1-S0; S0-S21-S3-S2-S0; & S0-S3-S0; S0-S39-S38-S0; S0-S37-S0; \\
\hline & & S0-S10-S0; S0-S24-S14-S0; & S0-S34-S0; S0-S33-S15-S0; S0-S33-S15-S0; \\
\hline \multirow{9}{*}{3} & \# routes: 14; Total frequencies: 15 ; & \# routes: 21 ; Total frequencies: 23 ; & \# routes: 29 ; Total frequencies: $30 ;$ \\
\hline & S0-S13-S0; S0-S19-S7-S0; & S0-S11-S0; S0-S24-S0; S0-S10-S19-S0; & S0-S21-S24-S27-S0; S0-S8-S0; S0-S4-S23-S0; S0-S9-S0; \\
\hline & $\underline{\text { S0-S14-S8-S12-S0; }}$ S0-S15-S2-S0; & S0-S17-S0; S0-S4-S21-S0; S0-S2-S0; & S0-S12-S0; S0-S39-S0; S0-S3-S0; S0-S32-S0; \\
\hline & S0-S16-S0; S0-S1-S9-S0; S0-S11-S0; & 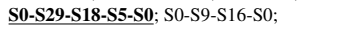 & S0-S18-S0; S0-S35-S0; S0-S11-S0; S0-S40-S31-S0; \\
\hline & S0-S5-S20-S0; S0-S18-S0; S0-S4-S0; & S0-S13-S8-S15-S0; S0-S20-S0; S0-S1-S0; & S0-S19-S15-S0; S0-S10-S0; S0-S28-S0; \\
\hline & S0-S17-S0; S0-S6-S0; S0-S10-S0; & S0-S30-S0; S0-S6-S0; S0-S7-S0; & S0-S22-S0; S0-S14-S0; S0-S17-S0; S0-S13-S0; \\
\hline & S0-S3-S0; & S0-S23-S0; S0-S26-S25-S0; S0-S28-S0; & S0-S30-S25-S34-S0; S0-S26-S0; S0-S16-S0; \\
\hline & & S0-S14-S0; S0-S3-S0; S0-S22-S27-S0; & S0-S5-S36-S7-S0; S0-S38-S1-S0; S0-S20-S0; \\
\hline & & S0-S12-S0; & S0-S37-S0; S0-S6-S0; S0-S2-S0; S0-S33-S29-S0; \\
\hline \multirow{9}{*}{4} & \# routes: 14 ; Total frequencies: 15 & \# routes: 21 ; Total frequencies: $21 ;$ & \# routes: $29 ;$ Total frequencies: 31 \\
\hline & S0-S13-S6-S0; S0-S15-S0; S0-S18-S0; & S0-S10-S28-S0; S0-S30-S0; S0-S25-S0; & S0-S9-S0; S0-S39-S0; S0-S16-S0; S0-S12-S0; \\
\hline & S0-S20-S0; $\underline{\text { S0-S17-S1-S8-S0; }}$ & S0-S4-S0; S0-S6-S29-S27-S0; S0-S26-S0; & S0-S19-S25-S0; S0-S27-S26-S0; S0-S28-S40-S0; \\
\hline & S0-S14-S0; S0-S12-S0; S0-S11-S2-S0; & S0-S18-S0; S0-S8-S0; S0-S23-S0; & S0-S17-S0; S0-S35-S0; S0-S7-S0; S0-S6-S0; \\
\hline & S0-S3-S0; S0-S19-S0; S0-S5-S9-S0; & S0-S24-S0; S0-S5-S0; S0-S14-S0; & S0-S23-S0; S0-S30-S0; S0-S32-S0; S0-S22-S0; \\
\hline & S0-S4-S0; S0-S10-S7-S0; S0-S16-S0; & S0-S11-S22-S0; S0-S9-S0; S0-S13-S0; & S0-S8-S24-S11-S0; S0-S2-S31-S33-S0; S0-S36-S0; \\
\hline & & S0-S21-S7-S0; S0-S3-S0; S0-S12-S0; & S0-S18-S0; S0-S1-S0; S0-S15-S3-S0; S0-S34-S14-S0; \\
\hline & & S0-S2-S19-S16-S0; S0-S17-S1-S0; & S0-S38-S0; S0-S10-S0; S0-S5-S0; S0-S4-S0; \\
\hline & & S0-S20-S15-S0; & 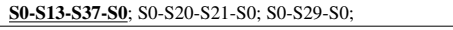 \\
\hline \multirow{9}{*}{5} & \# routes: $14 ;$ Total frequencies: 15 & \# routes: $21 ;$ Total frequencies: $22 ;$ & \# routes: 29 ; Total frequencies: 30 \\
\hline & S0-S13-S6-S0; S0-S15-S0; S0-S18-S0; & S0-S4-S26-S0; S0-S24-S0; S0-S17-S0; & S0-S10-S17-S0; S0-S14-S0; S0-S31-S0; S0-S1-S0; \\
\hline & S0-S20-S0; $\underline{\text { S0-S17-S1-S8-S0; }}$ & S0-S15-S0; S0-S20-S0; S0-S3-S2-S0; & S0-S27-S0; S0-S13-S0; S0-S20-S0; S0-S2-S0; \\
\hline & S0-S14-S0; S0-S12-S0; S0-S11-S2-S0; & S0-S30-S0; S0-S11-S6-S0; S0-S7-S0; & S0-S35-S0; S0-S11-S0; S0-S15-S0; S0-S28-S0; \\
\hline & S0-S3-S0; S0-S19-S0; S0-S5-S9-S0; & S0-S5-S27-S0; S0-S9-S0; S0-S10-S0; & S0-S3-S0; S0-S23-S0; S0-S16-S18-S0; S0-S36-S21-S0; \\
\hline & S0-S4-S0; S0-S10-S7-S0; S0-S16-S0; & S0-S8-S0; S0-S19-S0; S0-S12-S0; & S0-S9-S0; S0-S30-S26-S34-S0; S0-S24-S5-S6-S0; \\
\hline & & S0-S29-S0; S0-S22-S23-S0; S0-S1-S0; & S0-S37-S4-S0; S0-S40-S32-S0; S0-S12-S0; \\
\hline & & S0-S13-S0; S0-S21-S14-S16-S0; & S0-S22-S0; S0-S25-S7-S0; S0-S38-S29-S0; \\
\hline & & $\underline{\text { S0-S28-S18-S25-S0; }}$ & S0-S8-S0; S0-S19-S0; S0-S33-S0;S0-S39-S0; \\
\hline \multirow{9}{*}{6} & \# routes: 14; Total frequencies: 14 & \# routes: 21; Total frequencies: 24; & \# routes: $29 ;$ Total frequencies: 31 \\
\hline & S0-S4-S3-S0; S0-S18-S0; S0-S6-S0; & S0-S6-S0; S0-S13-S0; S0-S8-S0; & S0-S7-S0; S0-S6-S0; S0-S8-S0; S0-S34-S0; \\
\hline & S0-S17-S10-S0; S0-S8-S0; S0-S11-S0; & S0-S17-S7-S0; S0-S24-S0; S0-S23-S0; & S0-S18-S31-S37-S27-S22-S0 $;$ S0-S10-S0; S0-S30-S0; \\
\hline & S0-S20-S0; S0-S12-S16-S0; & S0-S10-S1-S27-S0; $\underline{\text { S0-S28-S15-S0; }}$ & S0-S3-S0; S0-S9-S0; S0-S26-S25-S0; S0-S12-S0; \\
\hline & S0-S19-S2-S0; S0-S9-S0; S0-S14-S0; & S0-S12-S0; S0-S4-S0; S0-S30-S0; & S0-S33-S0; S0-S29-S32-S0; S0-S28-S0; \\
\hline & S0-S1-S13-S5-S0; S0-S7-S0; & S0-S16-S29-S0; S0-S26-S0; S0-S11-S0; & S0-S24-S0; S0-S13-S0; S0-S39-S0; S0-S14-S0; \\
\hline & S0-S15-S0; & S0-S22-S0; S0-S25-S5-S0; $\underline{\text { S0-S9-S18-S0; }}$ & S0-S19-S0; S0-S23-S38-S0; S0-S20-S0; S0-S16-S0; \\
\hline & & 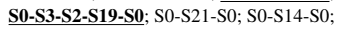 & S0-S21-S17-S2-S0; S0-S40-S36-S0; S0-S11-S0; \\
\hline & & S0-S20-S0; & S0-S15-S0; S0-S35-S0; S0-S4-S5-S0; S0-S1-S0; \\
\hline \multirow{9}{*}{7} & \# routes: 14 ; Total frequencies: 14 & \# routes: 21 ; Total frequencies: 22 ; & \# routes: $29 ;$ Total frequencies: 31 \\
\hline & S0-S10-S0; S0-S18-S0; S0-S20-S0; & S0-S11-S0; S0-S30-S0; S0-S8-S0; & S0-S35-S0; S0-S9-S28-S0; S0-S32-S0; S0-S34-S0; \\
\hline & S0-S16-S0; S0-S1-S0; S0-S14-S0; & S0-S2-S0; S0-S27-S29-S0; S0-S10-S0; & S0-S22-S0; S0-S8-S0; S0-S11-S0; S0-S13-S0; \\
\hline & S0-S3-S13-S0; S0-S8-S12-S0; & S0-S6-S0; S0-S13-S9-S0; S0-S24-S0; & S0-S26-S2-S0; S0-S30-S0; S0-S5-S23-S27-S0; \\
\hline & S0-S7-S0; S0-S19-S5-S0; S0-S17-S0; & S0-S28-S0; S0-S7-S17-S0; S0-S4-S0; & S0-S3-S0; S0-S25-S0; S0-S17-S0; S0-S19-S0; \\
\hline & S0-S11-S4-S9-S0; S0-S2-S0; & S0-S1-S0; S0-S12-S0; S0-S19-S0; & S0-S39-S0; S0-S33-S0; S0-S24-S0; S0-S38-S0; \\
\hline & S0-S15-S6-S0; & S0-S25-S5-S22-S0; S0-S18-S0; S0-S21-S0; & S0-S21-S40-S0; S0-S10-S0; S0-S15-S16-S0; \\
\hline & & S0-S15-S14-S0; S0-S23-S16-S0; & S0-S14-S0; S0-S1-S31-S0; S0-S20-S12-S0; S0-S4-S0; \\
\hline & & $\underline{\text { S0-S3-S26-S20-S0; }}$ & S0-S36-S7-S0; $\underbrace{\text { S0-S18-S37-S29-S0; }}$ S0-S6-S0; \\
\hline \multirow{8}{*}{8} & \# routes: 14; Total frequencies: 14; & \# routes: $21 ;$ Total frequencies: 21 & \# routes: $29 ;$ Total frequencies: $29 ;$ \\
\hline & S0-S3-S4-S2-S0; S0-S14-S0; & S0-S18-S0; S0-S27-S21-S0; S0-S13-S0; & S0-S13-S0; S0-S28-S0; S0-S12-S0; S0-S12-S0; \\
\hline & S0-S7-S20-S5-S0; S0-S8-S0; & S0-S10-S0; S0-S16-S0; S0-S5-S7-S0; & S0-S25-S20-S24-S0; S0-S4-S3-S0; S0-S39-S0; \\
\hline & S0-S10-S0; S0-S6-S0; S0-S1-S0; & S0-S1-S0; S0-S9-S0; S0-S6-S0; S0-S29-S0; & S0-S19-S0; S0-S27-S23-S0; S0-S29-S0; S0-S1-S0; \\
\hline & S0-S12-S0; S0-S11-S0; & S0-S25-S20-S23-S2-S0; S0-S8-S0; & S0-S34-S38-S0; S0-S37-S0; S0-S15-S0; S0-S8-S0; \\
\hline & S0-S16-S19-S0; S0-S18-S17-S0; & S0-S24-S19-S0; S0-S12-S0; S0-S17-S0; & S0-S11-S0; S0-S7-S0; S0-S9-S0; S0-S16-S0; \\
\hline & S0-S9-S0; S0-S13-S0; S0-S15-S0; & S0-S4-S3-S0; S0-S14-S0; S0-S30-S28-S0; & S0-S17-S26-S31-S0; S0-S6-S0; S0-S21-S0; S0-S10-S0; \\
\hline & & & $\begin{array}{l}\text { S0-S18-S0; S0-S14-S0; S0-S35-S0; S0-S5-S0; } \\
\text { S0-S22-S2-S33-S36-S0; S0-S32-S40-S0; S0-S30-S0; }\end{array}$ \\
\hline
\end{tabular}

Note: the frequencies with empty, underline, and underbrace symbols are 1,2, and 3, respectively. 
From Tables 4 and 5, we can see that when $n=20,30$ and 40, the corresponding routing decisions of the two models we obtained are quite different. In order to ensure the timely supply of parts from suppliers without generating any earliness and tardiness costs, the total transportation frequencies of the zero-inventory model is much higher than the ones of the P-LANE model. In addition, in the zero-inventory model, the transport frequency of each route is low during each production cycle, with a highest value of 3 . The reason may be that: in the zero-inventory model, Factory M's one-day production is divided into 8 production cycles, in which each of them will be independently planned, and the average demand of parts from each supplier is only one-eighth of the corresponding demand in the P-LANE model, thus the number of frequencies for each production cycle will be reasonably small values.

Figures 8 and 9 show the solution convergence of the P-LANE and the zero-inventory models, respectively. From these two figures, we can see that when the genetic algorithm is used to solve the minimum cost, as the number of iterations increases, the cost function value decreases continuously. When the number of iterations reaches about 100 , the convergence speed becomes slower and the minimum cost value tends to be stable.
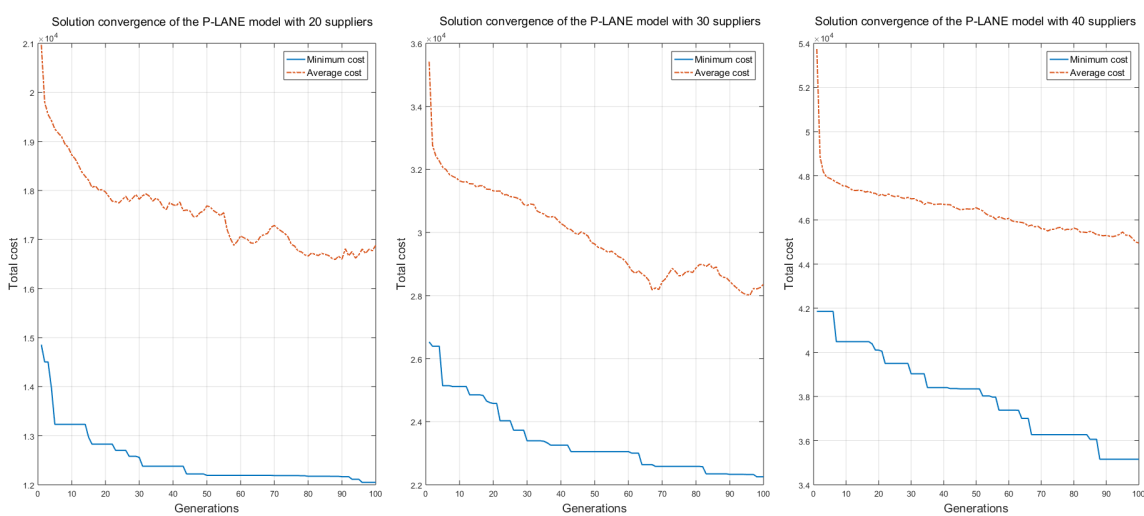

Fig. 8 The solution convergence of the P-LANE model with 20/30/40 suppliers

Table 6 shows the results of the total costs for the large-scale instances, in which the bold value indicates the optimal value of the category. We can see that the advantages of the P-LANE model become more obvious when the number of suppliers increases.

Table 6 Results of the costs for large-scale instances

\begin{tabular}{lllllll}
\hline \# Suppliers & Model & Transportation cost & Fixed cost & Earliness cost & Tardiness cost & Total costs \\
\hline \multirow{2}{*}{20} & P-LANE model & $\mathbf{8 2 1 4}$ & $\mathbf{9 7 2}$ & 1233 & 1628 & $\mathbf{1 2 0 4 7}$ \\
& Zero-inventory model & 14581 & 2070 & 0 & 0 & 16651 \\
\hline \multirow{2}{*}{30} & P-LANE model & $\mathbf{1 3 4 0 4}$ & $\mathbf{1 4 9 4}$ & 2655 & 4702 & $\mathbf{2 2 2 5 5}$ \\
& Zero-inventory model & 25450 & 3186 & $\mathbf{0}$ & $\mathbf{0}$ & 28636 \\
\hline \multirow{2}{*}{40} & P-LANE model & $\mathbf{2 0 9 6 5}$ & $\mathbf{2 1 0 6}$ & 4496 & 7590 & $\mathbf{3 5 1 5 7}$ \\
& Zero-inventory model & 41832 & 4392 & $\mathbf{0}$ & $\mathbf{0}$ & 46224 \\
\hline
\end{tabular}



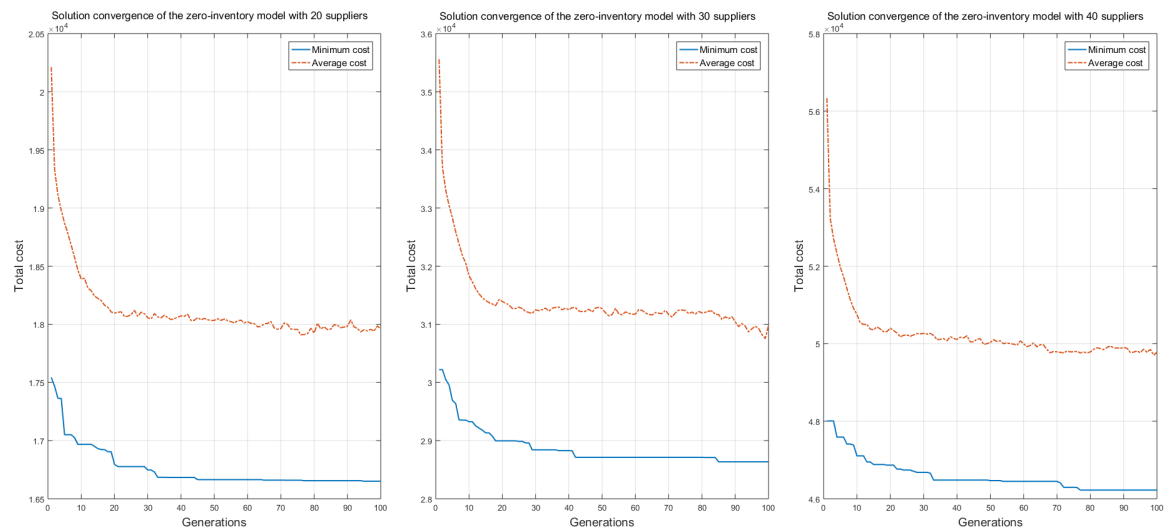

Fig. 9 The solution convergence of the zero-inventory model with 20/30/40 suppliers

\subsection{Comparison and discussion}

Table 7 summarizes the total frequency and the cost results obtained by the two models for both small- and large-scale instances. For small-scale instances, the total costs of the zeroinventory model are about $10.1 \%, 10.7 \%$ and $10.0 \%$ higher than the ones obtained by the PLANE model when the number of suppliers are 5, 6, and 7, respectively. For the large-scale instances, the total costs of the zero-inventory model are about $38.2 \%, 28.7 \%$ and $31.5 \%$ higher than the ones obtained by the P-LANE model when the number of suppliers are 20, 30 and 40 respectively. In addition, the transport frequency of the zero-inventory model is much higher than the one of the P-LANE model (e.g., more than twice the frequency when $n \geq 20$ ). Therefore, although joining with P-LANE increases the earliness and tardiness costs, the corresponding transportation and fixed costs could be significantly decreased, and thus results in a considerable reduction on the total costs.

Table 7 Comparison of the frequency and cost between two models

\begin{tabular}{llllllll}
\hline \multirow{2}{*}{ \# Suppliers } & Model & $\begin{array}{l}\text { Total } \\
\text { frequency }\end{array}$ & $\begin{array}{l}\text { Transportation } \\
\text { cost }\end{array}$ & $\begin{array}{l}\text { Fixed } \\
\text { cost }\end{array}$ & $\begin{array}{l}\text { Earliness } \\
\text { cost }\end{array}$ & $\begin{array}{l}\text { Tardiness } \\
\text { cost }\end{array}$ & $\begin{array}{l}\text { Total } \\
\text { costs }\end{array}$ \\
\hline \multirow{3}{*}{5} & P-LANE model & 17 & 3246 & 306 & 0 & 0 & 3552 \\
& Zero-inventory model & 20 & 3552 & 360 & 0 & 0 & 3912 \\
& Difference & $+3(17.6 \%)$ & +306 & +54 & 0 & 0 & $+360(10.1 \%)$ \\
\hline \multirow{3}{*}{6} & P-LANE model & 19 & 3726 & 342 & 400 & 0 & 4468 \\
& Zero-inventory model & 24 & 4512 & 432 & 0 & 0 & 4944 \\
& Difference & $+5(26.3 \%)$ & +786 & +90 & -400 & 0 & $+476(10.7 \%)$ \\
\hline \multirow{3}{*}{7} & P-LANE model & 20 & 4131 & 360 & 280 & 480 & 5251 \\
& Zero-inventory model & 24 & 5346 & 432 & 0 & 0 & 5778 \\
& Difference & $+4(20.0 \%)$ & +1215 & +72 & -280 & -480 & $+527(10.0 \%)$ \\
\multirow{3}{*}{20} & P-LANE model & 54 & 8214 & 972 & 1233 & 1628 & 12047 \\
& Zero-inventory model & 115 & 14581 & 2070 & 0 & 0 & 16651 \\
& Difference & $+61(113.0 \%)$ & +6367 & +1098 & -1233 & -1628 & $+4604(38.2 \%)$ \\
\hline \multirow{3}{*}{30} & P-LANE model & 83 & 13404 & 1494 & 2655 & 4702 & 22255 \\
& Zero-inventory model & 177 & 25450 & 3186 & 0 & 0 & 28636 \\
& Difference & $+94(113.2 \%)$ & +12046 & +1692 & -2655 & -4702 & $+6381(28.7 \%)$ \\
\hline \multirow{3}{*}{40} & P-LANE model & 117 & 20965 & 2106 & 4496 & 7590 & 35157 \\
& Zero-inventory model & 243 & 41832 & 4392 & 0 & 0 & 46224 \\
& Difference & $+126(107.7 \%)$ & +20867 & +2286 & -4496 & -7590 & $+11067(31.5 \%)$ \\
\hline
\end{tabular}


Figure 10 shows the total costs and the transport frequency of the zero-inventory and the P-LANE models for different scale instances. The left Y-axis represents the total costs while the right $\mathrm{Y}$-axis represents the total frequency. When the company uses the zeroinventory model, a very high milk-run frequency have to be used to collect automobile parts. Otherwise, the company may face a lack of the raw materials, and even cause a production line to shut down, which obviously increases the out-of-stock risks as well as high set-up costs. Meanwhile, it also cause a waste of truck capacity and incurs high total costs even though there is no earliness and tardiness costs in the factory.
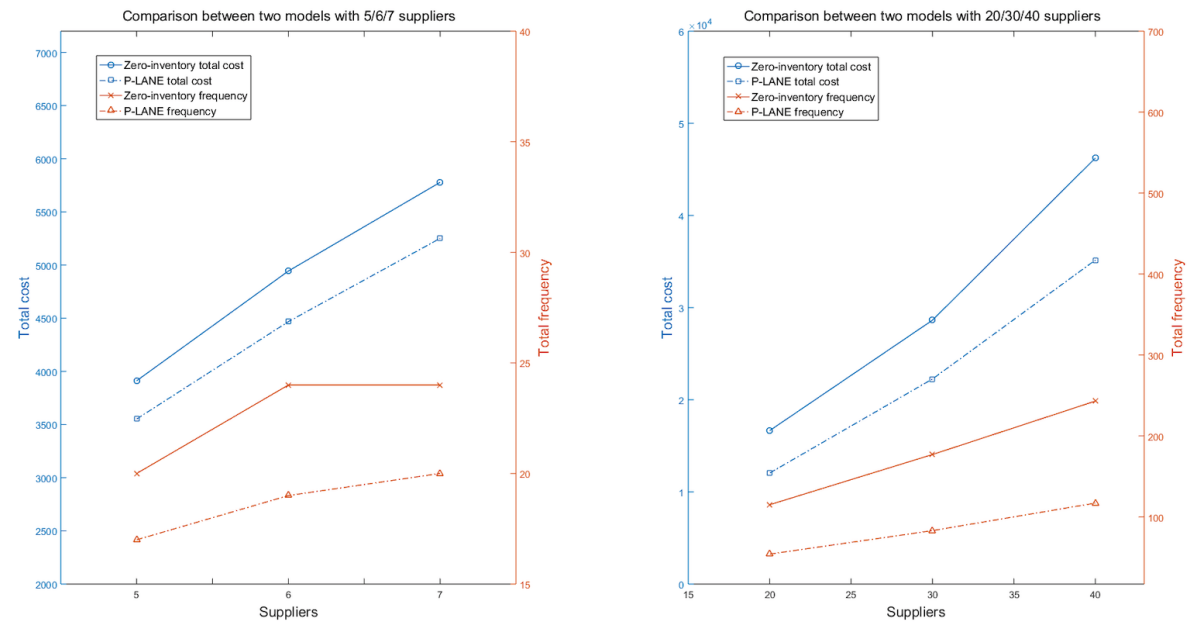

Fig. 10 Comparison of the total costs and transport frequency between two models for small- and large-scale instances

The above results show that the P-LANE model is superior to the zero-inventory model in terms of the total costs and the transport frequency. The main reason is that the proposed P-LANE model, which allows a reasonable inventory of raw materials, could flexibly adjust the transportation and production to meet the fluctuations in the daily production. Besides, in this paper, we assume that there are enough trucks to meet the zero-inventory model for high-frequency collection, but in practice, the number of truck is usually limited. Therefore, the P-LANE model proposed in this paper is more realistic and reasonable in real life, in which the parts achieve high turnover and inventory cost is not large but can act as a buffer.

Above all, in a practical production environment, it is recommended that logistics practitioners adopt the P-LANE model because it considers operational feasibility under the constraints of a time-based production system and demand fluctuation. It is also suggested that the proposed milk-run method with P-LANE can play an important role in the development of lean production and lean logistics.

\section{Conclusions}

Increasingly more manufacturers, especially automotive companies, are endeavoring to save costs. In addition, more organizations are improving inbound logistic activities and strategies to comply with low carbon and green development policies. Considering these trends, 
this paper studied the automobile parts collection problem considering the P-LANE model, which is a new storage policy used in the Toyota factory and has a significant effect on cost savings. Meanwhile, the current P-LANE scheduling within Toyota were designed based on expert experiences and lack a mathematic basis for calculation. The defined MRPPL studied in this paper differs from most previous VRP problems in which the P-LANE model is first taken into consideration and a mixed integer linear programming model is proposed.

The main contributions of this paper are as follows: 1) A new definition of a vehicle routing problem with P-LANE called MRPPL is considered, which is inspired by a real-world application and is a new direction for further VRP study: this research makes a theoretical contribution that advances the application of P-LANE by integrating an academic aspect to its practical aspect. 2) Modeling the MRPPL with mixed integer linear programming: so far, no study has investigated vehicle routing planning and transportation scheduling under the P-LANE model. 3) The development of a genetic algorithm for the MRPPL problem for large-scale instances. 4) Comparison of the P-LANE and zero-inventory models demonstrates the advantages and practical usefulness of the proposed P-LANE: the results show that the P-LANE significantly decreases total costs. 5) The case study on Factory M can be further extended to other companies, even they may belong to different industries, e.g., some specialist logistics companies may also use such methods to schedule the distribution routes and time windows.

A limitation of this research is that the number of P-LANEs is prefixed. Although this number comes from practical operations, future studies should use it as decision variable to obtain the optimal number of P-LANEs. In addition, in future studies on the MRPPL, hybrid algorithms combining the characteristics of exact and heuristic algorithms should be developed to improve the solution. Besides, to further demonstrate the advantage of using P-LANE in practical production, some hypotheses included in the modeling could be eliminated in future studies.

Acknowledgements The authors are grateful to the anonymous referees for their constructive comments and suggestions.

\section{References}

M. Alnahhal, A. Ridwan, and B. Noche. In-plant milk run decision problems. Facilities, 35 (22):25-45, 2014.

A. M. Benjamin and J. E. Beasley. Metaheuristics for the waste collection vehicle routing problem with time windows, driver rest period and multiple disposal facilities. Computers \& Operations Research, 37(12):2270-2280, 2010.

O. Berman and Q. Wang. Inbound logistic planning: Minimizing transportation and inventory cost. Transportation Science, 40(3):287-299, 2006.

B. Bilgen and I. Ozkarahan. Strategic tactical and operational production-distribution models: A review. International Journal of Technology Management, 28(2):151-171, 2004.

N. Boysen, S. Emde, M. Hoeck, and M. Kauderer. Part logistics in the automotive industry: Decision problems, literature review and research agenda. European Journal of Operational Research, 242(1):107-120, 2015.

J. Bramel and D. Simchi-Levi. A location based heuristic for general routing problems. Operations Research, 43(4):649-660, 1995.

G. S. Brar and G. Saini. Milk run logistics: Literature review and directions. In Proceedings of the World Congress on Engineering, volume 1, pages 6-8, 2011. 
C. Chen, Z. Tian, and B. Yao. Optimization of two-stage location-routing-inventory problem with time-windows in food distribution network. Annals of Operations Research, pages $1-24,2017$.

K. H. Chuah and J. C. Yingling. Routing for a just-in-time supply pickup and delivery system. Transportation Science, 39(3):328-339, 2005.

T. Du, F. K. Wang, and P.-Y. Lu. A real-time vehicle-dispatching system for consolidating milk runs. Transportation Research Part E: Logistics and Transportation Review, 43(5): 565-577, 2007.

A. El Fallahi, C. Prins, and R. W. Calvo. A memetic algorithm and a tabu search for the multi-compartment vehicle routing problem. Computers \& Operations Research, 35(5): 1725-1741, 2008.

A. Federgruen, M. Queyranne, and Y.-S. Zheng. Simple power-of-two policies are close to optimal in a general class of production/distribution networks with general joint setup costs. Mathematics of Operations Research, 17(4):951-963, 1992.

G. Gallego and D. Simchi-Levi. On the effectiveness of direct shipping strategy for the one-warehouse multi-retailer r-systems. Management Science, 36(2):240-243, 1990.

S. Gao, L. Qi, and L. Lei. Integrated batch production and distribution scheduling with limited vehicle capacity. International Journal of Production Economics, 160:13-25, 2015.

M. Gendreau, F. Guertin, J.-Y. Potvin, and R. Séguin. Neighborhood search heuristics for a dynamic vehicle dispatching problem with pick-ups and deliveries. Transportation Research Part C: Emerging Technologies, 14(3):157-174, 2006.

T. Henke, M. G. Speranza, and G. Wäscher. The multi-compartment vehicle routing problem with flexible compartment sizes. European Journal of Operational Research, 246(3): 730-743, 2015.

S. D. Hosseini, M. A. Shirazi, and B. Karimi. Cross-docking and milk run logistics in a consolidation network: A hybrid of harmony search and simulated annealing approach. Journal of Manufacturing Systems, 33(4):567-577, 2014.

Z. Jiang, Y. Huang, and J. Wang. Routing for the milk-run pickup system in automobile parts supply. In Proceedings of the 6th CIRP-Sponsored International Conference on Digital Enterprise Technology, pages 1267-1275. Springer, 2010.

H. S. Kilic, M. B. Durmusoglu, and M. Baskak. Classification and modeling for in-plant milk-run distribution systems. The International Journal of Advanced Manufacturing Technology, 62(9-12):1135-1146, 2012.

R. Lahyani, L. C. Coelho, M. Khemakhem, G. Laporte, and F. Semet. A multi-compartment vehicle routing problem arising in the collection of olive oil in tunisia. Omega, 51:1-10, 2015.

S. D. Lapierre and A. B. Ruiz. Scheduling logistic activities to improve hospital supply systems. Computers \& Operations Research, 34(3):624-641, 2007.

S. D. Lapierre, A. B. Ruiz, and P. Soriano. Designing distribution networks: Formulations and solution heuristic. Transportation Science, 38(2):174-187, 2004.

K. Li, Z.-H. Jia, and J. Y.-T. Leung. Integrated production and delivery on parallel batching machines. European Journal of Operational Research, 247(3):755-763, 2015.

J. R. Montoya-Torres, J. L. Franco, S. N. Isaza, H. F. Jiménez, and N. Herazo-Padilla. A literature review on the vehicle routing problem with multiple depots. Computers \& Industrial Engineering, 79:115-129, 2015.

T. Nemoto, K. Hayashi, and M. Hashimoto. Milk-run logistics by Japanese automobile manufacturers in Thailand. Procedia Social and Behavioral Sciences, 2(3):5980-5989, 2010. 
P. K. Nguyen, T. G. Crainic, and M. Toulouse. Multi-trip pickup and delivery problem with time windows and synchronization. Annals of Operations Research, 253(2):899-934, 2017.

D. Patel, M. B. Patel, and J. A. Vadher. Implementation of milk run material supply system in vehicle routing problem with simultaneous pickup and delivery. International Journal of Application or Innovation in Engineering \& Management, 3(11):122-124, 2014.

W. W. Qu, J. H. Bookbinder, and P. Iyogun. An integrated inventory-transportation system with modified periodic policy for multiple products. European Journal of Operational Research, 115(2):254-269, 1999.

S. J. Sadjadi, M. Jafari, and T. Amini. A new mathematical modeling and a genetic algorithm search for milk run problem (an auto industry supply chain case study). The International Journal of Advanced Manufacturing Technology, 44(1-2):194, 2009.

A. M. Sarmiento and R. Nagi. A review of integrated analysis of production-distribution systems. IIE Transactions, 31(11):1061-1074, 1999.

P. Sitek and J. Wikarek. Capacitated vehicle routing problem with pick-up and alternative delivery (CVRPPAD): Model and implementation using hybrid approach. Annals of Operations Research, pages 1-21, 2017.

T. Staab, E. Klenk, S. Galka, and W. A. Günthner. Efficiency in in-plant milk-run systems The influence of routing strategies on system utilization and process stability. Journal of Simulation, 10(2):137-143, 2016.

E.-G. Talbi. Metaheuristics: From design to implementation, volume 74. John Wiley \& Sons, 2009. 
Appendix

Table 8 Coordinates of the manufacturer and 40 suppliers converted from their longitudes and latitude values

\begin{tabular}{|c|c|c|c|c|}
\hline Manufacturer & Longitude & Latitude & $\mathrm{X}$ & $\mathrm{Y}$ \\
\hline Factory M (S0) & 117.738896 & 39.063279 & 563.9522906 & 4325.865383 \\
\hline Supplier & Longitude & Latitude & $\mathrm{X}$ & $\mathrm{Y}$ \\
\hline S1 & 117.7202 & 39.06129 & 562.3332 & 4325.632 \\
\hline S2 & 117.3529 & 39.06881 & 530.5392 & 4326.279 \\
\hline S3 & 117.3527 & 38.95679 & 530.5704 & 4313.843 \\
\hline S4 & 117.3527 & 38.95679 & 530.5704 & 4313.843 \\
\hline S5 & 117.4402 & 39.13335 & 538.06 & 4333.477 \\
\hline S6 & 117.7043 & 39.06515 & 560.9599 & 4326.049 \\
\hline S7 & 117.3243 & 39.25007 & 527.9958 & 4346.393 \\
\hline S8 & 117.7842 & 39.21503 & 567.7292 & 4342.746 \\
\hline S9 & 117.7164 & 39.06777 & 561.9974 & 4326.348 \\
\hline S10 & 117.7079 & 39.06998 & 561.2615 & 4326.588 \\
\hline S11 & 117.7019 & 39.07698 & 560.7424 & 4327.362 \\
\hline $\mathrm{S} 12$ & 117.7019 & 39.07698 & 560.7424 & 4327.362 \\
\hline S13 & 117.7196 & 39.08346 & 562.2601 & 4328.092 \\
\hline S14 & 117.7102 & 39.07899 & 561.4524 & 4327.589 \\
\hline S15 & 117.7819 & 39.20647 & 567.5416 & 4341.793 \\
\hline S16 & 117.6928 & 39.07862 & 559.9467 & 4327.537 \\
\hline S17 & 117.5645 & 39.08969 & 548.8389 & 4328.69 \\
\hline S18 & 117.4136 & 39.13849 & 535.7571 & 4334.037 \\
\hline S19 & 117.4202 & 39.13762 & 536.3276 & 4333.942 \\
\hline S20 & 117.2483 & 39.23677 & 521.4336 & 4344.895 \\
\hline S21 & 117.4013 & 38.82949 & 534.8491 & 4299.728 \\
\hline S22 & 117.3678 & 39.08268 & 531.8215 & 4327.823 \\
\hline S23 & 117.357 & 39.06996 & 530.8954 & 4326.407 \\
\hline S24 & 117.1862 & 39.13322 & 516.102 & 4333.387 \\
\hline S25 & 117.2521 & 39.23846 & 521.7678 & 4345.085 \\
\hline S26 & 117.2472 & 39.18306 & 521.3579 & 4338.933 \\
\hline S27 & 117.4587 & 38.99195 & 39.7403 & 4317.781 \\
\hline S28 & 117.7065 & 39.05448 & 561.1556 & 4324.866 \\
\hline S29 & 117.7292 & 39.09067 & 563.0844 & 4328.9 \\
\hline S30 & 117.7135 & 39.05321 & 561.7641 & 4324.73 \\
\hline S31 & 117.256 & 38.98357 & 522.1806 & 4316.787 \\
\hline S32 & 117.2604 & 38.9812 & 522.568 & 4316.525 \\
\hline S33 & 117.2564 & 38.99206 & 522.2141 & 4317.73 \\
\hline S34 & 117.2414 & 39.0186 & 520.9096 & 4320.673 \\
\hline S35 & 117.7391 & 39.08273 & 563.9506 & 4328.024 \\
\hline S36 & 117.2744 & 38.98376 & 523.7776 & 4316.814 \\
\hline S37 & 117.2604 & 38.9812 & 522.568 & 4316.525 \\
\hline S38 & 117.2112 & 38.98066 & 518.3043 & 4316.454 \\
\hline S39 & 117.2876 & 38.90221 & 524.9503 & 4307.763 \\
\hline S40 & 117.266 & 38.99569 & 523.0401 & 4318.135 \\
\hline
\end{tabular}

